\title{
War die Politik 1877 weitsichtiger als 2018?
}

\author{
Christoph Hänggeli \\ Rechtsanwalt, Geschäftsführer des Schweizerischen Instituts für ärztliche Weiter- und Fortbildung SIWF
}

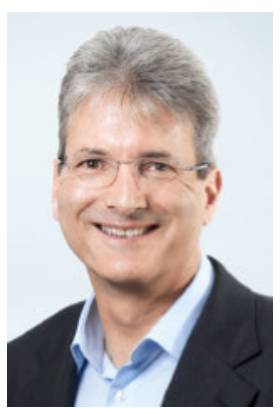

Mit der per 1.1.2018 in Kraft getretenen Revision des Medizinalberufegesetzes (MedBG) sind in der Schweiz zwei wichtige Anliegen für den Patientenschutz realisiert worden: Einerseits müssen alle Ärztinnen und Ärzte vor Aufnahme einer beruflichen Tätigkeit ihr Diplom überprüfen und im offiziellen Medizinalberuferegister (MedReg) eintragen lassen. Andererseits müssen sie die für die jeweilige Berufsausübung notwendigen Sprachkenntnisse nachweisen [1]. Damit gehören «vermeintliche» Ärzte, welche ohne oder mit gefälschten Diplomen Patienten gefährden, der Vergangenheit an. Dank des öffentlich zugänglichen Registers können Patientinnen und Patienten jederzeit prüfen, ob die sie behandelnden Ärztinnen und Ärzte über die notwendigen Diplome verfügen.

Auch die Spitäler sind bei der Rekrutierung ausländischer Fachkräfte von der schwierigen Gleichwertigkeitsbeurteilung der Diplome befreit und dürfen sich auf den rechtsverbindlichen Registereintrag verlassen. Einen Fortschritt stellen auch die «notwendigen Kenntnisse einer Amtssprache des Kantons» dar, welche für die Berufsausübungsbewilligung gefordert sind. Fehldiagnosen und falsche Therapieentscheidungen aufgrund von fehlendem Sprachverständnis sollten also nicht mehr vorkommen.

Dank des offiziellen Registers können Patienten jederzeit die Diplome ihrer behandelnden Ärztinnen und Ärzte überprüfen.

Folglich alles paletti - Ziel erreicht? Jein. Ein Wermutstropfen bleibt: Bereits kurz nach der Inkraftsetzung der Revision haben einige Kantone ein seltsames Verständnis bezüglich der geforderten Sprachkompetenzen an den Tag gelegt. So hat beispielsweise der Kanton Tessin einem Zürcher Arzt mit eidgenössischem Arztdiplom die Anstellung in einem Tessiner Spital verwehrt mit der Begründung, dass er nicht über die er- forderlichen Sprachkenntnisse verfüge, obwohl er als Ehemann einer Tessinerin fliessend italienisch spricht. Seit 1877 garantieren sowohl die Bundesverfassung wie auch das Freizügigkeitsgesetz, dass Inhaber des eidgenössischen Arztdiploms zur freien Berufsausübung im Gebiete der ganzen Eidgenossenschaft befugt sind. Eine Errungenschaft und ein Paradebeispiel weitsichti-

Inhaber des eidgenössischen Arztdiploms sollen auch weiterhin in der ganzen Schweiz ihren Beruf ausüben können.

ger Politik aus dem vorletzten Jahrhundert! Und seit 2018 soll diese Freizügigkeit plötzlich nicht mehr gelten? Die Medizinalberufeverordnung (MedBV) kennt in Art. 11c tatsächlich nur drei Kriterien für den Eintrag der Sprachkenntnisse im Register: ein höchstens sechs Jahre altes international anerkanntes Sprachdiplom, ein in der entsprechenden Sprache erworbener Ausoder Weiterbildungsabschluss oder Arbeitserfahrung als Arzt in der entsprechenden Sprache im Umfang von drei Jahren. Die im schweizerischen Maturzeugnis ausgewiesenen Sprachkenntnisse sind offensichtlich vergessen gegangen. Der Gesetzgeber war wohl kaum der Meinung, dass beispielsweise eine Tessinerin, welche nach der Matura ihr Medizinstudium in Zürich absolviert hat, nicht zur Weiterbildung in den Kanton Tessin zurückkehren darf - mangels Kenntnis der italienischen Sprache ... Ebenso grotesk wäre der Entscheid, wenn ein Arzt mit Matur und Studium im Kanton Bern anschliessend in Lausanne oder Genf keine Praxis eröffnen dürfte. Immerhin hat sich das für das MedBG zuständige BAG bereit erklärt, diese unbefriedigende Situation zu überprüfen. Es würde nicht von Weitsicht zeugen, wenn die seit 1877 garantierte Freizügigkeit für eidgenössisch diplomierte Ärztinnen und Ärzte 140 Jahre später einem Versehen auf Verordnungsstufe zum Opfer fiele. 